\title{
A new hypothesis for how sex steroid hormones regulate bone mass
}

\author{
Joseph Lorenzo
}

The University of Connecticut Health Center, Farmington, Connecticut, USA

\begin{abstract}
It has been proposed - but remains controversial - that estrogen's effects on various tissues may be mediated by different cell signaling pathways. Researchers have identified (see related article beginning on page 1651) a synthetic ligand that activates only a subset of these pathways, suggesting that bypass of the traditional estrogen pathway can prevent bone loss without associated side effects on reproductive organs.
\end{abstract}

J. Clin. Invest. 111:1641-1643 (2003). doi:10.1172/JCI200318812.

Sex steroid hormones exert a variety of important actions. In addition to their role as regulators of reproductive functions, they have potent effects on the nervous and cardiovascular system and are major determinants of the development and the structural integrity of the skeleton. Estrogens and androgens regulate such diverse cellular actions as proliferation, morphogenesis, differentiation, and apoptosis, or programmed cell death. However, our understanding of the pathways by which sex steroid hormones influence cellular functions is incomplete.

The classical mechanism of steroid hormone action involves interactions with intracellular receptors (Figure 1), which are either cytoplasmic or nuclear $(1,2)$. Subsequently, the receptor-ligand complex binds to specific cis-active elements of the promoter regions in the DNA of responsive genes. A key role of sex steroid hormones in the regulation of gene transcription is the recruitment of a complex of coactivators and corepressors to the receptor-ligand-DNA binding site. This is true for estrogen receptors (ERs) $\alpha$ and $\beta$ as well as the

\footnotetext{
Address correspondence to: Joseph Lorenzo, The University of Connecticut Health Center, Division of Endocrinology L3104, MC-1317, Farmington, Connecticut 06030-1317, USA. Phone: (860) 679-8199; Fax: (860) 679-1040; E-mail: jlorenzo@nso2.uchc.edu.

Conflict of interest: The author has declared that no conflict of interest exists.

Nonstandard abbreviations used: estrogen receptor (ER); androgen receptor (AR); selective estrogen receptor modulator (SERM); extracellular signal-regulated kinase (ERK).
}

androgen receptor (AR). It is now appreciated that the relative balance of receptors, coactivator, and corepressor proteins is a critical determinant of the ability of this classical pathway to initiate responses. Since the relative concentrations of these molecules is cell specific, sex steroid hormones can have vastly different functions in different tissues of the same organism. Variations in the recruitment of coregulatory molecules also appear to be the mechanism by which selective estrogen receptor modulators (SERMs) produce their tissue-specific effects (3).

A second mechanism of action for the classic pathway involves proteinprotein interactions (Figure 1). Typically, in this pathway, receptor-ligand complexes interact with transcription factors to alter the ability of the transcription factor to influence gene transcription. An example of this mechanism is the capacity of ligatedER complexes to influence the function of activator protein-1 and specific protein-1 $(4,5)$. However, a common characteristic of both of these classic pathways is that changes in gene transcription typically occur after 30-60 minutes.

In contrast, it is now appreciated that more rapid nonclassical pathways of sex steroid hormone action allow both estrogens and androgens to influence cellular function. Typically, these effects occur within seconds to minutes. In 1967, Szego and Davis (6) demonstrated that $17 \beta$-estradiol given intravenously increased uterine $3^{\prime}, 5^{\prime}$-cyclic adenosine monophosphate (cAMP) levels within 15 seconds. Pietras and Szego (7) found that the outer surface of endometrial cells contained estrogen-binding sites. In recent years it has become apparent that rapid effects of steroid hormones are mediated by interactions with components of various signal transduction pathways, including adenylyl cyclase, MAPKs, and PI3K.

Interestingly, the receptors that bind sex steroid ligands and initiate these responses can be identical to or different from known steroid receptors (Figure 1). Estrogen can bind the G protein-coupled receptor homolog GPR30 and activate extracellular signal-regulated kinases (ERKs) 1 and 2 in SK-BR-3 breast cancer cells (8). ERK-1 and ERK-2 are members of the MAPK family. Significantly, SK-BR-3 cells are devoid of both ER $\alpha$ and ER $\beta$. Activation of ERKs by estrogen in SK-BR-3 cells was not prevented by an ER antagonist, which blocks binding of estrogen to ER (8).

Estrogen is also capable of inducing rapid effects via membrane-bound ERs. Typically, the majority of either $E R \alpha$ or ER $\beta$ is found in the nucleus. However, small amounts (2\%) can associate with the cell membrane. Accumulation of sex steroid hormone receptors in the cell membrane is patchy, suggesting the presence of structures that are critical for their localization. Both ER $\alpha$ and $\operatorname{ER} \beta$ as well as AR can accumulate in caveolae, which are 50- to 100-nm flask-shaped subcompartments in the cell membrane that contains the $22-\mathrm{kDa}$ transmembrane phosphoprotein caveolin. After binding ligand, ERs or ARs in the cell membrane can interact with c-Src and activate MAPK pathways $(1,9)$.

In earlier investigations, Kousteni and colleagues examined the role of estrogen in preventing the bone loss that develops relatively rapidly in mice after sex steroid hormones are withdrawn $(10,11)$. In this issue of the JCI, Kousteni et al. (12) now present data that link the rapid activation of MAPK by nonclassical pathways to the ability of sex steroid hormones to regulate apoptosis in bone cells. 


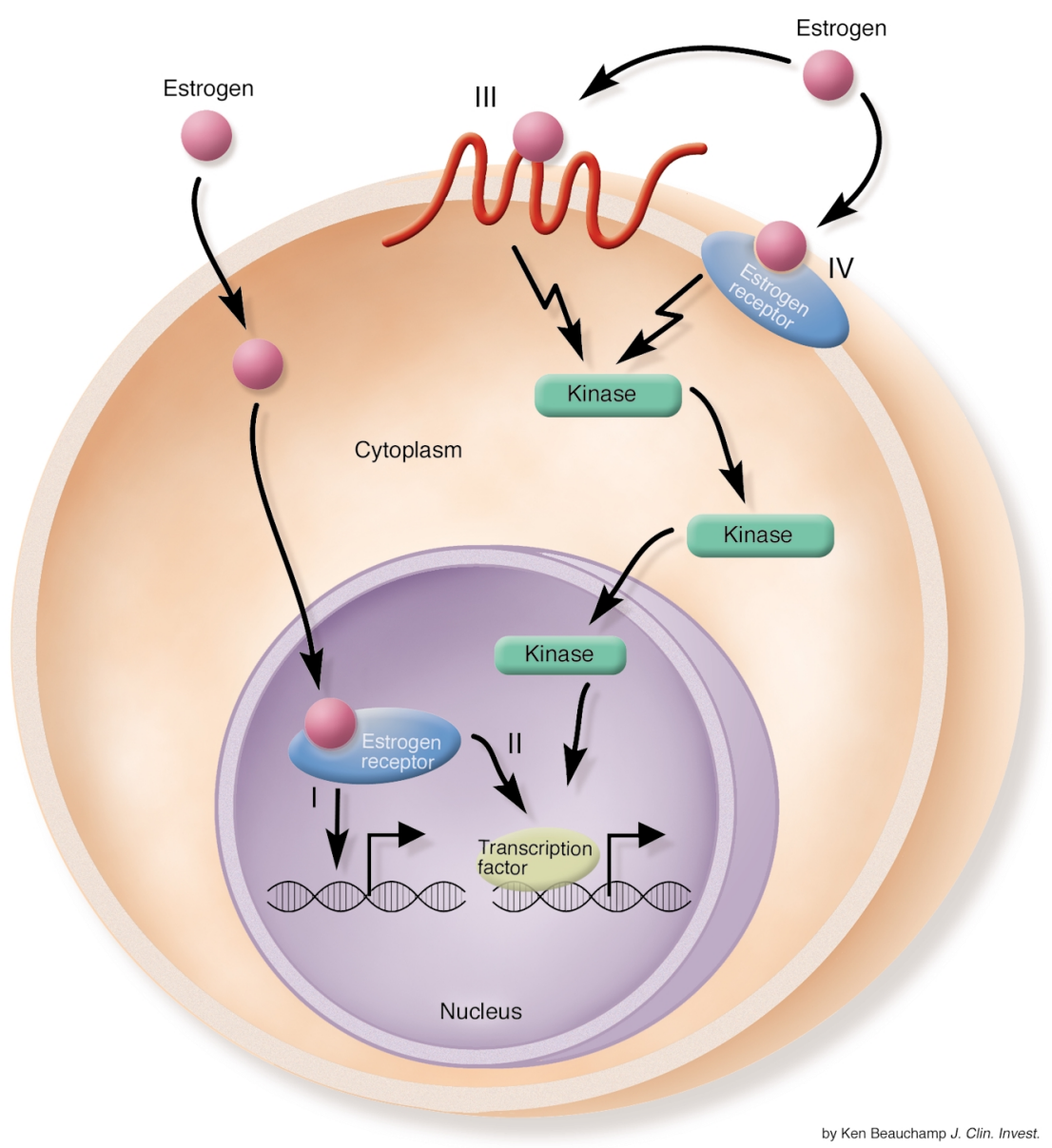

Kousteni et al. (9) previously demonstrated in cell culture that the ability of estrogens or androgens to regulate apoptosis was mediated by the ligandbinding domain of sex steroid hormone receptors that were localized exclusively to the cell membrane. This response was seen in HeLa cells transfected with ERs or ARs and in osteoblast and osteocyte cell culture models. The ability of sex steroid hormones to regulate apoptosis in these models was not specific for a particular sex steroid hormone receptor, since estrogens or androgens were equally effective in mediating responses in HeLa cells transfected with either ERs or ARs. The authors hypothesized that a major effect of sex steroid hormones in preventing bone loss results from inhibition of apoptosis in osteoblasts and stimulation of apoptosis in osteoclasts.

In the current study (12), the authors use a series of dominant negative analogs of components of the Src, PI3K, ERK, and JNK pathways to demonstrate the critical role of these signaling mechanisms in mediating the ability of sex steroid hormones to block apoptosis in cell culture models. In addition, key to their studies is the use of the synthetic ligand 4-estren$3 \alpha, 17 \beta$-diol, which the authors refer to as estren. This compound showed no classical sex steroid hormone activity in the examined cell culture systems. However, estren was a potent activator of the rapid cell membrane-mediated Src-MAPK pathways in cell culture models. Furthermore, it blocked apoptosis in osteoblast models and stimulated apoptosis in osteoclast models. In contrast, raloxifene - a SERM currently approved to treat osteoporosis - did not mimic the apoptosis-regulating effects of estren in cell culture models. In addition, studies published last year by these authors (11) demonstrated that estren, at concentrations 300 -fold higher than those of $17 \beta$ estradiol, blocked the bone loss that occurred after ovariectomy in a mouse model. Based on the results of both their previous work $(9,11)$ and the cur-

\section{Figure 1}

Sex steroid hormones can affect cellular function by a variety of mechanisms. The illustration depicts the mechanisms by which estrogen influences cells. The classical pathways (I and II) depend on direct interaction of estrogen with its receptor in the nucleus. Once activated, the estrogen-receptor complex can directly mediate gene transcription (I) or interact with transcription factors (II) to influence their activity. The nonclassical pathways (III and IV) work more rapidly and depend on the ability of estrogen to interact with either nonsteroid hormone receptors (III) or steroid hormone receptors in the membrane (IV). Both nonclassical pathways activate kinases that ultimately regulate transcription of specific genes. Adapted with permission from ref. 16 . rent study (12), the authors postulate that estren has a different mechanism of action than do SERMs, since the latter exclusively use the classical pathways to regulate gene transcription in a cell-specific manner. The authors believe, based on their in vitro data, that estren influences cellular function through its ability to influence cell membrane-mediated nonclassical responses to sex steroid hormones.

This is a provocative and controversial hypothesis that requires additional investigation before it can be fully accepted. The skeleton is a complex organ system that is regulated by the interactions of both bone-forming osteoblasts (derived from mesenchymal precursor cells) and bone-resorbing osteoclasts (derived from hematopoietic precursors). Compounding the complexity of the signals that regulate bone homeostasis are interactions with marrow cells, which are in close association to bone, and the responses of bone cells to the myriad cytokines that are produced in the bone micro- 
environment. The relatively simple cell culture systems that Kousteni et al. (9, 12) have examined to date are unlikely to mimic the complex interactions that regulate the skeleton in vivo. It also is unclear whether the effects of sex steroid hormone withdrawal on the skeleton are mediated predominantly by the ability of these agents to regulate the differentiation of osteoblasts and osteoclasts from their precursor cells or, as Kousteni et al. postulate (12), the ability of sex steroid hormones to regulate apoptosis. There is now strong evidence in murine models that estrogen withdrawal is associated with an increased number of osteoclast precursor cells in the marrow (10), an effect that has been linked to the regulation of B-lymphopoiesis (13-15). Since the target cell for this response is unknown, it is unclear what pathways estren or estrogen uses to produce this response. Hence, studies of the mechanism by which sex steroid hormones or estren mediate their bone-sparing effects ultimately will require in vivo experiments that use sophisticated technologies to dissect in detail both the cell-specific and the subcellular compartment-specific effects that these agents have on bone mass. Once data from such in vivo studies become available, a more complete understanding of the many effects that sex steroid hormones have on the skeleton should be appreciated.

1. Cato, A.C., Nestl, A., and Mink, S. 2002. Rapid actions of steroid receptors in cellular signaling pathways. Sci. STKE. 138:RE9.

2.Shupnik, M.A. 2002. Oestrogen receptors, receptor variants and oestrogen actions in the hypothalamic-pituitary axis. J. Neuroendocrinol. 14:85-94.

3. Riggs, B.L., and Hartmann, L.C. 2003. Selective estrogen-receptor modulators: mechanisms of action and application to clinical practice. N. Engl. J. Med. 348:618-629.

4. Paech, K., et al. 1997. Differential ligand activation of estrogen receptors ERalpha and ERbeta at AP1 sites. Science. 277:1508-1510.

5. Saville, B., et al. 2000. Ligand-, cell-, and estrogen receptor subtype (alpha/beta)-dependent activation at GC-rich (Sp1) promoter elements. J. Biol. Chem. 275:5379-5387.

6. Szego, C.M., and Davis, J.S. 1967. Adenosine 3',5' monophosphate in rat uterus: acute elevation by estrogen. Proc. Natl. Acad. Sci. U. S. A. 58:1711-1718.

7. Pietras, R.J., and Szego, C.M. 1977. Specific bind- ing sites for oestrogen at the outer surfaces of isolated endometrial cells. Nature. 265:69-72.

8. Filardo, E.J., Quinn, J.A., Bland, K.I., and Frackelton, A.R., Jr. 2000. Estrogen-induced activation of Erk-1 and Erk-2 requires the G protein-coupled receptor homolog, GPR30, and occurs via transactivation of the epidermal growth factor receptor through release of HB-EGF. Mol. Endocrinol. 14:1649-1660

9. Kousteni, S., et al. 2001. Nongenotropic, sex-non specific signaling through the estrogen or androgen receptors: dissociation from transcriptional activity. Cell. 104:719-730.

10. Jilka, R.L., et al. 1992. Increased osteoclast development after estrogen loss: mediation by interleukin-6. Science. 257:88-91.

11. Kousteni, S., et al. 2002. Reversal of bone loss in mice by nongenotropic signaling of sex steroids. Science. 298:843-846.

12. Kousteni, S., et al. 2003. Kinase-mediated regulation of common transcription factors accounts for the bone-protective effects of sex steroids. J. Clin. Invest. 111:1651-1664. doi:10.1172/JCI200317261.

13. Manabe, N., et al. 2001. Connection between B lymphocyte and osteoclast differentiation pathways. J. Immunol. 167:2625-2631.

14. Sato, T., Shibata, T., Ikeda, K., and Watanabe, K. 2001. Generation of bone-resorbing osteoclasts from B220+ cells: its role in accelerated osteoclastogenesis due to estrogen deficiency. J. Bone Miner. Res. 16:2215-2221.

15. Katavic, V., et al. 2003. The surface antigen CD45R identifies a population of estrogen-regulated murine marrow cells that contain osteoclast precursors. Bone. In press.

16. Miller, G. 2002. Divorcing estrogen's bright and dark sides. Science. 298:723-724.

\section{What is the real role of antimicrobial polypeptides that can mediate several other inflammatory responses?}

\section{Peter Elsbach}

Department of Medicine, New York University School of Medicine, New York, New York, USA

Antimicrobial peptides are effector molecules of innate immunity with microbicidal and pro- or anti-inflammatory activities. Their role is now widening following evidence that one such multifunctional peptide, LL-37, induces angiogenesis, a process essential for host defense, wound healing, and tissue repair (see related article beginning on page 1665).

J. Clin. Invest. 111:1643-1645 (2003). doi:10.1172/JCI200318761.

The last two decades have heralded impressive progress in the identification of a broad array of structurally and functionally diverse polypeptides

\footnotetext{
Address correspondence to: Peter Elsbach, Department of Medicine, New York University School of Medicine, 550 First Avenue, New York, New York 10016, USA. Phone: (212) 263-5633; Fax: (212) 263-3952; E-mail: elsbap01@mcrcr.med.nyu.edu. Conflict of interest: The author has declared that no conflict of interest exists.
}

implicated in many aspects of the host response to infection and other inflammatory stimuli.

Almost half a century ago, Hirsch described the antimicrobial properties of phagocytin, a crude protein fraction of polymorphonuclear leukocytes (1), thereby setting the stage for a growing interest in the biologic activities of protein components of inflammatory cells. The subsequent exploration of the functional capabilities of defined proteins and peptides in inflammation paralleled the evolving methodology of protein biochemistry and purification, and molecular biology. In this historical context, the initial focus remained on the antibacterial action of newly isolated proteins and peptides, reflecting an ongoing search for new antibiotics and the relative ease of conducting bioassays of bacterial viability $(2,3)$.

\section{Discovery of proteins and polypeptides with antibacterial properties}

Numerous proteins and polypeptides with antimicrobial activity in vitro have now been isolated and/or cloned from a broad range of both simple and complex organisms, including humans. Thus, endogenous-polypeptide antibiotics became prominent targets in the study of antimicrobial host defenses per se and also as potential pharmacologic agents (3). Whole families of such gene products have been identified in plants, insects, and other animals (4) in settings that are consistent with prominent roles in innate immunity. How well have these roles been defined so far? 